\title{
Mindfulness and Autonomous Sensory Meridian Response (ASMR)
}

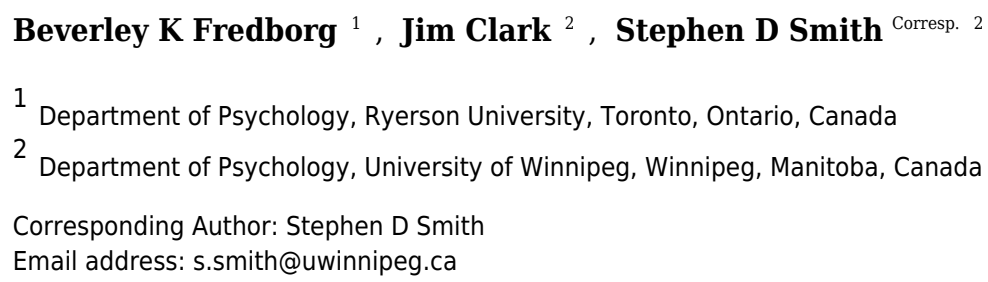

Background: Autonomous Sensory Meridian Response (ASMR) is a perceptual phenomenon in which specific audiovisual stimuli frequently elicit tingling sensations on the scalp and neck. These stimuli ("ASMR triggers") are typically social in nature (e.g., watching someone brush their hair, hearing whispering,) and often elicit a calm and positive emotional state that may last up to several minutes.ASMR experiences phenomenologically overlap with mindfulness; however, no research has directly examined how mindfulness might relate to ASMR. Methods: In the current study, 284 individuals with ASMR completed the Toronto Mindfulness Scale (TMS), the Mindful Attention and Awareness Scale (MAAS), and a questionnaire examining ASMR experiences. Age- and sexmatched control participants were asked to view two ASMR-eliciting videos to ensure that they did not experience tingling sensations associated with ASMR; they then completed the TMS and MAAS questionnaires. Results: When compared with matched controls, individuals with ASMR generated significantly higher scores on the Mindful Attention and Awareness Scale, a global measure of mindfulness, as well as significantly higher scores on the Curiosity subscale of the Toronto Mindfulness Scale. Conclusions: These results suggest that the sensory-emotional experiences associated with ASMR may be partially explained by a distinct subset of characteristics associated with mindfulness. 
$7{ }^{1}$ Department of Psychology, Ryerson University, 350 Victoria Street, Toronto, Ontario, Canada,

$8 \quad M 5 B 2$ K3. Email: bfredborg@psych.ryerson.ca

$9{ }^{2}$ Department of Psychology, University of Winnipeg, 515 Portage Avenue, Winnipeg, Manitoba,

10 Canada, R3B 2E9. Phone: (204) 786-9757. Email: j.clark@uwinnipeg.ca

$11{ }^{3}$ Department of Psychology, University of Winnipeg, 515 Portage Avenue, Winnipeg, Manitoba,

12 Canada, R3B 2E9. Phone: (204) 786-9737. Email: s.smith@uwinnipeg.ca

*Corresponding Author

\section{Paper Statistics}

16 Word count (excluding table and references): 4724

17 Figures: 0

18 Tables: 2

19 References: 17 


\section{Abstract}

25

26

27

Background: Autonomous Sensory Meridian Response (ASMR) is a perceptual phenomenon in which specific audiovisual stimuli frequently elicit tingling sensations on the scalp and neck. These stimuli ("ASMR triggers") are typically social in nature (e.g., watching someone brush their hair, hearing whispering,) and often elicit a calm and positive emotional state that may last up to several minutes. ASMR experiences phenomenologically overlap with mindfulness; however, no research has directly examined how mindfulness might relate to ASMR. Methods: In the current study, 284 individuals with ASMR completed the Toronto Mindfulness Scale (TMS), the Mindful Attention and Awareness Scale (MAAS), and a questionnaire examining ASMR experiences. Age- and sex-matched control participants were asked to view two ASMReliciting videos to ensure that they did not experience tingling sensations associated with ASMR; they then completed the TMS and MAAS questionnaires. Results: When compared with matched controls, individuals with ASMR generated significantly higher scores on the Mindful Attention and Awareness Scale, a global measure of mindfulness, as well as significantly higher scores on the Curiosity subscale of the Toronto Mindfulness Scale. Conclusions: These results suggest that the sensory-emotional experiences associated with ASMR may be partially explained by a distinct subset of characteristics associated with mindfulness. 

specific stimuli, known as "ASMR triggers", frequently elicit tingling sensations on the scalp, neck, and shoulders, often spreading to the body's periphery. These sensations are generally accompanied by a sense of calm as well as by positive affect (Barratt \& Davis, 2015). The triggers that elicit these experiences vary widely between individuals and may be auditory, visual, tactile, and/or olfactory in nature. Despite their heterogeneity, the most popular ASMR triggers tend to be audio-visual; a recent survey study of individuals with ASMR found that whispering and close-up attention elicited tingles in over half of the 450 respondents (Barratt \& Davis, 2015).

Although the phenomenology of ASMR overlaps with other atypical conscious states, there are elements of the ASMR experience that appear to be unique. For instance, unlike other sensory-emotional experiences such as frisson (i.e., "chills" elicited by music or an emotional experience), which tend to last up to 10 seconds, individuals with ASMR may experience the tingling sensations induced by a triggering stimulus for several minutes or more (del Campo \& Kehle, 2016; Fredborg, Clark, \& Smith, 2017). In addition, the intensity and duration of ASMR experiences are often under the control of the individual, a characteristic that distinguishes ASMR from the automatic sensory responses of synesthesia. Indeed, the ability to suppress ASMR responses as they arise may be an important aspect of this phenomenon that warrants further study. Another noteworthy quality of ASMR is that the tingles tend to induce relaxation and positive affect in the individual. The fact that ASMR is associated with both atypical sensory experiences and an emotional response suggests that in order to fully understand ASMR, it will be necessary to examine other conditions or experiences that produce similar emotional and sensory changes. 
64

65

66

67

68

One conscious state that shares some phenomenological characteristics with ASMR is mindfulness (Barratt \& Davis, 2015). In their operationalization of mindfulness, Bishop and colleagues (2004) define the construct as a two-component process by which one engages in both intentional self-regulation of attention and a nonjudgmental awareness and acceptance of the present moment. Other researchers have suggested that mindfulness involves an openness to sensations, attentional control, emotional regulation, and resilience (Kabat-Zinn, 1990). Both of these complementary descriptions of mindfulness overlap with elements of the ASMR experience. For example, the focused attention method of mindfulness meditation requires individuals to focus on a specific external stimulus or internal thought (Lutz, Slagter, Dunne, \& Davidson, 2008). During ASMR experiences, individuals focus attention on an external stimulus that triggers tingling sensations. Both mindfulness and ASMR can lead to a feeling of relaxation that enhances people's subjective well-being (Barratt \& Davis, 2004; Bishop et al., 2004). Given these similarities, an examination of the relationship between ASMR and mindfulness seems warranted.

The current research investigated the relationship between ASMR and both state and trait mindfulness. State mindfulness reflects a transient, moment-to-moment conscious experience (Tanay \& Bernstein, 2013) whereas trait mindfulness is a more stable and enduring tendency to attend to and experience the world in a mindful manner (Brown \& Ryan, 2003). Individuals with ASMR and matched control participants completed two well-established self-report measures of mindfulness-the Toronto Mindfulness Scale, which is more sensitive to state mindfulness (TMS; Lau et al., 2006), and the Mindful Attention and Awareness Scale, which is more sensitive to the attentional component of trait mindfulness (MAAS; Brown \& Ryan, 2003). ASMR participants also completed the ASMR Checklist (Fredborg, Clark, \& Smith, 2017), 
87 which examines ASMR triggers and the phenomenology of ASMR experiences. We predicted

88 that individuals with ASMR would report higher levels of mindfulness, as measured by both the TMS and the MAAS. We also predicted that individuals with ASMR who reported higher intensity tingles in response to common triggering stimuli would score higher on both the TMS and MAAS than individuals who reported lower intensity tingles. Together, these data would provide novel insight into the phenomenology of ASMR, while also showing how this atypical conscious state is related to mindful states and traits.

\section{Methods and Materials}

\section{Participants}

The current study is the second part of a two-part study that examined personality traits that co-occur with ASMR (Fredborg et al., 2017). Two-hundred eighty-four participants who self-reported as experiencing $\operatorname{ASMR}\left(n=135\right.$ males, $\left.M_{\text {age }}=28.07, S D=9.58\right)$ and 279 participants who did not indicate ever experiencing ASMR $\left(n=123\right.$ males, $M_{\text {age }}=29.19, S D=$ $10.55)$ took part in this study. Given the exploratory nature of this study, an a priori power analysis to determine the ideal sample size for a given effect size would have been based on speculation and was thus not conducted.

Recruitment of participants with ASMR was conducted via an advertisement for the study on a Reddit.com forum dedicated to ASMR (http://www.reddit.com/r/ASMR). As ASMR was largely discovered through discussion on internet forums (del Campo \& Kehle, 2016), we used this recruitment strategy to find as many individuals with ASMR as possible who would be willing to share their experiences online. To gain confidence that individuals recruited to the ASMR group genuinely experienced the phenomenon (rather than watched the videos for relaxation purposes), they were asked to answer specific questions about their ASMR 
110 experiences when completing a self-created ASMR checklist (Fredborg et al., 2017; as

111 elaborated below). These questions included a list of 14 popular ASMR-triggering stimuli that

112 participants rated both on the intensity of tingling sensations experienced, as well as how many

113 seconds after stimulus onset that they would typically experience tingles. Only participants who

114 completed the entirety of the ASMR checklist were included in the ASMR group during the

115 analyses.

116 Age- and sex-matched control participants were recruited by the participant recruitment

117 team at Qualtrics Panels (Qualtrics, Inc., Provo, UT) which is an internet-based participant

118 recruitment company. Using this recruitment method allowed us to mitigate sampling bias by

119 increasing the likelihood that both ASMR and control participants were regular internet users.

120 All control participants provided informed consent prior to study completion. To ensure that they

121 did not experience ASMR, control participants viewed two popular ASMR-eliciting videos and

122 were asked whether the videos triggered tingling sensations. If an individual indicated that they

123 had experienced ASMR either during the videos or in the past, he or she was guided to an exit

124 screen and thanked for their time. Further details of this screening method are included in the

125 Procedure section.

126 The current study received ethical approval from the University of Winnipeg Department

127 of Psychology’s Human Research Ethics Board (HREB).

\section{Questionnaires}

The survey included several well-established questionnaires: the Big Five Inventory

130 (BFI; John, Donahue, \& Kentle, 1991), the Mindful Attention and Awareness Scale (MAAS:

131 Brown \& Ryan, 2003) and the Toronto Mindfulness Scale (TMS: Lau, Bishop, Segal, Buis,

132 Anderson, \& Calson, 2006). It also included several self-created questionnaires: a self-created 
133 Embodied Emotion Scale, a self-created ASMR Checklist (Fredborg et al., 2017), and a set of

134 demographic questions to characterize the sample. The results of the relationship between the

135 Big Five personality traits and ASMR have been published separately (Fredborg et al., 2017).

136 The present report focuses on the TMS and the MAAS, and their relationship to data from the 137 ASMR Checklist.

138 The TMS consists of 13 self-report items that assess experiences of mindfulness

139 immediately preceding a meditation session (Lau et al., 2006). Individuals respond to each item

140 using a 5-point Likert-type scale from 0 ("not at all") to 4 ("very much"). The 13 items on the

141 TMS load onto two subscales. Six items comprise the Curiosity subscale of the TMS, which is a

142 measure of one's curiosity about any thoughts, emotions, and sensations that arise while

143 engaging in a mindful state. Examples of items on the Curiosity subscale include, "I was more

144 invested in just watching my experiences as they arose, than in figuring out what they could

145 mean," and, "I remained curious about the nature of each experience as it arose." The remaining

146 seven items comprise the Decentering subscale, which measures people's awareness of their own

147 experiences. Items on the Decentering subscale include, "I experienced myself as separate from

148 my changing thoughts and feelings," and, "I was aware of my thoughts and feelings without

149 overidentifying with them" (Lau et al., 2006). In its validation sample, both the Curiosity and

150 Decentering subscales of the TMS demonstrated good levels of internal consistency as measured

151 by Cronbach's alpha ( $\alpha=0.88$ and $\alpha=0.84$, respectively). The instructions to this questionnaire

152 were slightly modified to be suitable for individuals with ASMR experiences.

153

The MAAS consists of 15 self-report items that load onto a single trait mindfulness factor

154 (Brown \& Ryan, 2003). Items assess people's ability to attend to and be aware of the present

moment, and are rated using a 6-point Likert-type scale with response options ranging from 
156 "almost always" to "almost never." Examples of items, which are all reverse-coded, include, "I

157 could be experiencing some emotion and not be conscious of it until sometime later," and "I rush

158 through activities without being really attentive to them." Studies have reported that the MAAS

159 has strong psychometric properties; in its validation sample, the authors reported good internal

160 consistency as measured by Cronbach's alpha $(\alpha=.82)$ as well as high levels of convergent and

161 discriminant validity. Similarly, in their study of self-report measures of mindfulness, Baer,

162 Smith, Hopkins, Krietemeyer, and Toney (2006) reported a Cronbach's alpha value of .86 for

163 this measure.

The ASMR Checklist (Fredborg et al., 2017) consists of questions regarding the ASMR participants' experiences including the intensity of respondents' responses to 16 triggers that often elicit ASMR as well as the speed of onset of these tingling sensations. After initial analyses (as described in Fredborg et al., 2017), two of these 16 items were removed because only a very small proportion of the sample reported familiarity with these items. These stimuli items were "watching others sweep" and "watching others refill fountain pens". As such, 14 popular ASMRinducing stimuli were analyzed. Individuals were asked to rate each stimulus on a 7-point Likert scale from 0 to 6 (from "no tingles" to the "most intense ASMR experience."), or were provided the option to choose "unknown" if they were unsure if a stimulus elicited tingles. Additional questions examined the length of time it would take for an individual to experience

174 the tingling sensation after the onset of each of the 14 stimuli, the frequency with which respondents view videos that will elicit ASMR, whether they find ASMR experiences to be pleasurable, and whether they use ASMR videos to relax. 


\section{Procedure}

After completing an initial informed consent procedure on the Qualtrics survey, individuals who self-identified as having ASMR were asked to complete five questionnaires (TMS, MAAS, BFI, ASMR Checklist, and a self-created Embodied Emotion Scale) presented in an order that was randomized for each individual. Individuals could save their progress on the questionnaires and return later (within a two-week period) to complete the study should they need to take a break. After completing the five questionnaires, participants completed a set of standard demographic questions. Upon study completion, participants were thanked for their contribution to ASMR research, and were given the option to provide comments on the study. Control participants recruited through Qualtrics Panels similarly read and accepted the terms of an informed consent form before beginning the experiment. To ensure their inclusion in the control group, prospective control participants watched a minimum of five minutes of a popular ASMR video embedded in the survey, followed by two questions about their experiences both while watching the video and in daily life. These questions were, "Did you experience tingles while watching the video?" and, "Have you ever experienced intense pleasurable tingles in the head or neck while listening to or watching something mundane happening (for example, watching someone paint their nails or draw)?” If answered affirmatively, participants were guided to an exit screen and thanked for their time. Participants who did not report experiencing ASMR continued with the study by completing the same four questionnaires (TMS, MAAS, BFI, and a self-created Embodied Emotion Scale) presented in a randomized order, as well as the same set of demographic questions to characterize the sample. Participants in the control group did not complete the ASMR checklist. Upon study completion, Control participants were 
201 similarly thanked for their contribution to ASMR research, and were given the option to provide

202 comments on the study.

203

204

205

206

207

208

209

210

211

212

213

214

215

216

217

218

219

220

221

222

223

\section{Results}

The ASMR and Control groups were first compared on the TMS and the MAAS. These comparisons were followed by an analysis of the TMS and the MAAS results in relation to responses on the ASMR Checklist. All analyses were performed using SPSS Statistics for Windows, Version 19.0 (SPSS, Inc., Armonk, NY).

\section{Toronto Mindfulness Scale}

Scores for the Curiosity and Decentering subscales of the TMS were calculated per their standard scoring protocol (Lau et al., 2006; see Table 1 for descriptive statistics). Reliability analyses for each subscale were run within the ASMR and control groups, respectively. Although the internal consistency, as measured by Cronbach's alpha, was excellent for the Curiosity subscale across both groups (ASMR participants: $\alpha=.89$, Control participants: $\alpha=$ .93), poor reliability was found for the Decentering subscale for ASMR participants (ASMR participants: $\alpha=.60$, Control participants: $\alpha=.83$ ). Further item-by-item reliability analyses revealed that no single item was unfavorably skewing the internal consistency of the Decentering subscale.

Please Insert Table 1 Here

To further explore the poor reliability results of the Decentering subscale, and to adhere to recommendations to run exploratory factor analyses when using established test measures in new contexts (Flake, Pek, \& Hehman, 2017), a principle components factor analysis (PCA) with 
224 Varimax rotations was run on all 13 items of the TMS separately for ASMR and control

225 participants. For ASMR participants, the PCA revealed three factors with eigenvalues greater

226 than 1.0, which in total explained $57.5 \%$ of the variance. In contrast to its construction sample

227 (Lau et al., 2006), all Curiosity subscale items and one Decentering item (i.e., "I am more

228 concerned with being open to my experiences than controlling or changing them") loaded

229 together onto the first factor. The remaining Decentering subscale items were split into two new

230 factors: the first comprised of four items related to acceptance and awareness of thoughts (i.e., "I

231 am aware of my thoughts and feelings without over-identifying with them," "I approach each

232 experience by trying to accept it," "I am more invested in just watching my experiences as they

233 arise, than in figuring out what they could mean," and "I am receptive to observing unpleasant

234 thoughts and feelings without interfering with them"), while the second comprised of two items

235 related to viewing oneself as separate from thoughts (e.g., "I experience myself as separate from

236 my changing thoughts and feelings" and "I experience my thoughts more as events in my mind

237 than as a necessarily accurate reflections of the way things 'really' are"). As such, the poor

238 reliability results for the Decentering subscale may, in part, be explained by its split into two

239 distinct factors for ASMR participants.

240 Consistent with the construction sample, for control participants, a PCA revealed two

241 factors with eigenvalues greater than 1.0, which in total explained $61.8 \%$ of the variance. In

242 contrast, the first factor was comprised of all six Curiosity subscale items, as well as two

243 Decentering subscale items (i.e., "I am aware of my thoughts and feelings without

244 overidentifying with them," and "I approach each experience by trying to accept it, no matter if it

245 was pleasant or unpleasant"). The second factor was comprised of four remaining items from the

246 Decentering subscale. 
of the TMS is reported here, as all items on the Curiosity subscale loaded onto the primary factor of the TMS for both the ASMR and control participants.

As predicted, the ASMR group generated higher scores on the Curiosity subscale of the

TMS than did the Control group, $F(1,559)=12.935, M S E=36.456, p<0.001, \eta_{\mathrm{p}}{ }^{2}=0.023$.

252

There was no main effect of Gender on this subscale, indicating no overall differences between

Males and Females on Curiosity, $F(1,559)=1.478, M S E=.36 .456, p=0.225, \eta_{\mathrm{p}}{ }^{2}=0.003$.

However, the main effect of Group was qualified by a significant Group by Gender interaction, $F(1,559)=5.746, M S E=36.456, p=0.017, \eta_{\mathrm{p}}{ }^{2}=0.010$. Simple effects analyses revealed that for ASMR participants, Females $(M=14.98, S D=5.60)$ generated significantly higher scores than Males $(M=13.133, S D=5.69)$ on Curiosity, $F(1,559)=6.624, p=0.01, \eta_{\mathrm{p}}{ }^{2}=0.012$. For Control participants, there was no significant difference between Males and Females on this subscale, $p=.407$. Thus, our results indicate that females with ASMR have significantly higher levels of the Curiosity sub-facet of mindfulness than males with the condition.

\section{Mindful Attention Awareness Scale}

The total score on the MAAS was computed according to a standard scoring protocol (Brown \& Ryan, 2003). Consistent with previous research (e.g., Baer et al., 2006; Brown \& Ryan, 2003), reliability analyses revealed excellent internal consistency as measured by Cronbach's alpha (ASMR participants: $\alpha=.85$, Control participants: $\alpha=.95$ ). Overall, the MAAS and the TMS Curiosity subscale were modestly positively correlated, $r(563)=.234, p<$ 0.001. However, when analyzed by group (with the total sample of participants per group), the correlation between the MAAS and the TMS Curiosity subscale for the ASMR group was lower for ASMR participants, $r(284)=.132, p=.026$, than Control participants, $r(279)=.265, p<$ 
270

271

272

273

274

275

276

277

278

279

280

281

282

283

284

285

286

287

288

289

290

291

292

0.001. As predicted, the ASMR group generated higher scores on the MAAS than the Control group, $F(1,559)=15.423, M S E=.963, p<0.001, \eta_{\mathrm{p}}{ }^{2}=0.027$. There was no main effect of

Gender, $F(1,559)=.835, M S E=.963, p=.361, \eta_{\mathrm{p}}{ }^{2}=0.001$, nor a significant interaction between Group and Gender, $F(1,559)=.421, M S E=.963, p=.516, \eta_{\mathrm{p}}{ }^{2}=0.001$.

\section{ASMR Checklist and Mindfulness Measures}

As previously published (Fredborg et al., 2017), reliability analyses revealed good internal consistency for the ASMR Checklist, as indicated by a Cronbach's alpha of .81. A principal-components analysis of the 14 items on the checklist revealed five "intensity" factors underlying the common ASMR-triggering stimuli chosen for the checklist, each of which had an eigenvalue greater than 1.0. These intensity factors (with items in parentheses) were Watching ("Watching others paint," "Watching others draw," "Watching others open a package," and "Watching others cook"), Touching ("Watching someone touch another person's hair,"

"Watching someone touch their own hair," "Watching others apply makeup and/or nail polish to themselves," and "Watching others apply makeup and/or nail polish to another person"), Repetitive Sounds (“Tapping sounds" and "Scratching sounds”), Simulations ("Dentist simulation" and "Haircut simulation"), and Mouth Sounds ("Chewing sounds" and "Whispering"), respectively (Fredborg et al., 2017). The average intensity of tingles experienced by all ASMR participants $(\mathrm{N}=284)$ in response to the triggering stimuli on the ASMR checklist was calculated and was normally distributed, $M_{\text {Total }}=2.35, S D=1.01, D(284)=0.036$, NS.

Means and standard deviations for the 14 intensity items on this checklist are reported elsewhere (Fredborg et al., 2017, Table 2, p. 5).

Scores on the Curiosity subscale of the TMS and the MAAS were correlated with $M_{\text {Total }}$ and the five intensity factors. The overall relationship between $M_{\text {Total }}$ and the mindfulness 
293 measures was based on the total sample of ASMR participants $(N=284)$. Correlations between

294 the mindfulness measures and the five intensity factors were based only on the 173 ASMR

295 participants who provided numerical intensity ratings to all 14 stimuli (i.e., no stimuli were rated 296 as "unknown"). .

297 Consistent with the prediction that ASMR participants would score higher than controls 298 on the Curiosity subscale, intensity ratings ( $\left.M_{\text {Total }}\right)$ were positively correlated with Curiosity 299 subscale, $r(284)=.160, p=.007$. This effect appears to be primarily due to the "Touching", $300 r(173)=.200, p=.008$, and "Repetitive Sounds", $r(173)=.235, p=.002$, factors. Scores on the 301 Curiosity subscale were not significantly correlated with any other factor, $|r| \mathrm{s}<.09, p s>.24$. $M_{\text {Total }}$ intensity ratings also correlated positively with the MAAS, $r(284)=.112, p=.059$, although this result was only marginally significant. This effect is primarily due to the "Simulations" factor, $r(173)=.153, p=.044$. MAAS scores were not significantly correlated with any other factor, $|r| \mathrm{s}<.115, p \mathrm{~s}>.130$. See Table 2 for a list of the correlations between mindfulness scores and ASMR Checklist responses.

Additional ASMR Checklist Measures. Analyses were also performed on the ASMR

311 Checklist items unrelated to individuals' ASMR triggers. One item asked respondents to

312 indicate whether they experienced chills (i.e., frisson) and whether they were able to distinguish 313 between the phenomenological experiences of ASMR and frisson. The results clearly showed 314 that individuals with ASMR can distinguish between ASMR and frisson; of the 248 individuals 315 with ASMR who identified that they experience frisson (87.3\% of respondents with ASMR), 
316

317

318

320

321

322

323

324

325

326

327

328

329

330

331

332

333

334

335

336

337 338

90.7\% indicated that ASMR and frisson are distinct perceptual experiences. Furthermore, the experience of frisson within this sample is modestly positively correlated with mindful Curiosity as measured by the Curiosity subscale of the TMS, $r(284)=.131, p=0.027$.

The ASMR Checklist also asked respondents to indicate the degree to which ASMR tingles are experienced as pleasurable. On average, $76.1 \%$ of respondents indicated that ASMR experiences are "quite pleasurable" and $22.9 \%$ of respondents indicated that ASMR experiences are "mildly pleasurable." The remaining response options_-"neutral," "mildly uncomfortable," and "quite uncomfortable" - each received one response ( $0.4 \%$ of the total respondents).

Correlation analyses revealed that as ratings of ASMR pleasure increased, so did ratings of tingle intensity, $r(284)=.215, p<0.001$. Further, ratings of ASMR tingle pleasure were positively correlated with scores on the Curiosity subscale of the TMS, $r(284)=.146, p=0.014$.

Finally, ASMR respondents were asked to indicate how often, on average, they use ASMR-triggering videos to relax. Of the 284 ASMR participants surveyed, $5.3 \%$ of respondents indicated that they "never" use videos to relax, $6.7 \%$ indicated that they use them "less than once a month," 4.6\% indicated that they use them "one a month," $9.2 \%$ use them "2-3 times a month," $14.1 \%$ use them "once a week," $32.7 \%$ use them "2-3 times a week," and $27.5 \%$ use them

"daily." Correlation analyses revealed that the more often people use ASMR-triggering videos to relax, the more intense their tingling experiences, though this was only a modest correlation, $r(284)=.124, p=0.037$. Further, a modest positive correlation between the frequency of using ASMR-triggering videos to relax and scores on the Curiosity subscale on the TMS was revealed, though this effect was marginally nonsignificant, $r(284)=.114, p=0.055$. In contrast, there was no significant correlation between the frequency of using ASMR-triggering videos to relax and scores on the MAAS, $p=0.37$. 


\section{Discussion}

The current study strongly supports the hypothesis that ASMR is related to mindfulness.

341

342

343

344

345

346

347

348

349

350

351

352

353

354

355

356

357

358

359

360

361

Individuals with ASMR generated higher scores on the MAAS, a measure of the attentional component of mindfulness. They also produced higher scores on the Curiosity subscale of the

Toronto Mindfulness Scale, suggesting a greater interest in and openness to their own conscious experiences. This latter result is also consistent with previous research indicating that individuals with ASMR have higher levels of the openness-to-experience personality trait (Fredborg et al., 2017; Janik McErlean \& Banissy, 2017).

The results of the Decentering subscale of the TMS were less clear due to differences in reliability between the groups. Although the internal consistency for the control participants was congruent with previous research (Cronbach's $\alpha=.83$; Lau et al., 2006), the internal consistency for the ASMR participants was much lower (Cronbach's $\alpha=.60)$. This difference suggests that individuals in the ASMR group did not interpret the questions on this subscale of the TMS in the same way. Some participants may have interpreted these items in a more general (i.e., trait-like) fashion while others interpreted them as being related to the ASMR 'tingling' experience (i.e., a state response). Moreover, exploratory factor analyses of the TMS for ASMR participants revealed a three-factor structure in which the Decentering subscale was split into two factors.

Given these mixed results, it is problematic to compare the control group to the ASMR group on this subscale. However, the fact that individuals with ASMR reported higher levels of curiosity as well as increased mindful attention suggests that ASMR may be a cognitively 'active' process rather than a more automatic response to stimuli.

The current study is not the first to find inter-group differences in the interpretation of items in mindfulness scales. Williams and Dalgleish (2014) found that meditators and 
362 nonmeditators differ in their interpretation of some items on the Five-Facet Mindfulness

363 Questionnaire (FFMQ; Baer, Smith, Hopkins, Krietemeyer, \& Toney, 2006). These group

364 differences were largest for items relating to the "observe" facet of mindfulness, which focuses

365 on the observation of external and internal stimuli and includes such questions as, "When I'm

366 walking, I deliberately notice the sensations of my body moving." This component of the FFMQ

367 appears to overlap with the Decentering subscale of the TMS. Given that individuals with

368 ASMR are more mindful than controls, the notion that individuals with ASMR may be more

369 mindfully curious, but equally aware of their experiences, as controls is actually consistent with

370 previous research (e.g., Aguado, Luciano, Cebolla, Serrano-Blanco, Soler, \& Garcia-Campayo,

371 2015; Williams \& Dalgleish, 2014). Further research is necessary to determine whether

372 individuals with ASMR are more or less adept than controls at other facets of mindfulness, such

373 as decentering (e.g., the ability for people to view themselves as separate from their thoughts and

374 feelings).

375 Additional research is also necessary to characterize the sex differences found in our

376 study. Both male and female ASMR participants scored higher than controls on the MAAS, a

377 measure of the attentional component of ASMR. On the Curiosity subscale of the TMS,

378 however, females with ASMR generated higher scores than males with ASMR. Future studies

379 combining self-report measures with psychophysiological and/or neuroimaging methods would

380 help elucidate the reason(s) for these potential sex differences.

381 The current study also addressed the relationship between ASMR intensity, specific

382 ASMR triggers, and mindfulness. The overall ASMR intensity score, $M_{\text {Total }}$, was indeed

383 correlated with the Curiosity subscale of the TMS. This result suggests that individuals who are

384 curious about stimuli will experience ASMR more intensely than less-curious individuals. This, 
385

386

387

388

389

390

391

392

393

394

395

396

397

398

399

400

401

402

403

404

405

406

again, suggests that ASMR is a cognitively active process. The pattern of ASMR triggers also suggests that perceptual curiosity is a factor in ASMR experiences. The "Touching" and "Repetitive Sounds" triggers significantly correlated with Curiosity. It is possible that these types of triggers require more top-down cognitive interpretation than triggers such as whispering. Listening to repetitive sounds and watching people touch things may require the observer to create a mental structure of the perceived stimuli. Individuals who are proficient at doing so—or who have the motivation or curiosity to do so-would be more likely to experience tingles associated with these stimuli.

The current results raise an interesting question: Given that mindfulness is associated with increased ASMR intensity, would mindfulness training lead to enhanced ASMR experiences? The current data suggest that it would, although additional research is necessary to test this hypothesis. If such a study produced positive results, it would present individuals with ASMR with some interesting possibilities. Many individuals with ASMR enjoy 'tingles' hedonically or as a form of relaxation, whereas others use ASMR to help reduce the intensity of some of the symptoms associated with anxiety and depression (Barratt \& Davis, 2015). If mindfulness training were to enhance the subjective effects of ASMR, it is possible that this training might enhance any potential benefits that ASMR has on subjective well-being. However, these possible benefits of ASMR are, at present, speculative. To date, the positive impact of ASMR on mental and physical health has only been discussed anecdotally and in selfreport survey form. Future research investigating the clinical utility of ASMR, alone and in conjunction with other therapies, would help address this intriguing possibility.

\section{Conclusions}


410 ASMR, suggesting that the cognitive underpinnings of ASMR may differ between the sexes.

411 Future studies are necessary to thoroughly characterize these mechanisms. Additional research

412 is also necessary to determine whether ASMR could be used to enhance well-being in a manner 413 similar to many mindfulness-based treatment programs. 
415 The authors would like to acknowledge the staff at Qualtrics Panels for collecting participant

416 data from individuals who do not experience ASMR and for their willingness to provide

417 technical support on this project. Moreover, without the members of the ASMR Reddit

418 community, this study would not have been possible. 


\section{References}

Aguado, J., Luciano, J.V., Cebolla, A., Serrano-Blanco, A., Soler, J., \& Garcia-Campayo, J. (2015). Bifactor analysis and construct validity of the five facet mindfulness questionnaire (FFMQ) in non-clinical Spanish samples. Frontiers in Psychology, 6, 404. doi: 10.3389/fpsyg.2015.00404.

Baer, R. A., Smith, G. T., Hopkins, J., Krietemeyer, J., \& Toney, L. (2006). Using self-report assessment methods to explore facets of mindfulness. Assessment, 13, 27-45. doi: $10.1177 / 1073191105283504$

Barratt, E. L., and Davis, N. J. (2015). Autonomous Sensory Meridian Response (ASMR): a flow-like mental state. PeerJ., 3, e851. doi: 10.7717/peerj.851

Bishop, S. R., Lau, M., Shapiro, S., Carlson, L., Anderson, N. D., Carmody, J., ... \& Devins, G. (2004). Mindfulness: A proposed operational definition. Clinical psychology: Science and practice, 11, 230-241.

Brown, K. W., and Ryan, R. M. (2003). The benefits of being present: mindfulness and its role in psychological well-being. Journal of Personality and Social Psychology, 84, 822-848. doi: $10.1037 / 0022-3514.84 .4 .822$

Costa P. T., Jr., Terracciano, A., \& McCrae, R. R. (2001). Gender differences in personality traits across cultures: robust and surprising findings. Journal of personality and social psychology, 81, 322-331. doi: 10.1037//0022-3514.81.2.32

del Campo, M. A., \& Kehle, T. J. (2016). Autonomous sensory meridian response (ASMR) and frisson: Mindfully induced sensory phenomena that promote happiness. International Journal of School \& Educational Psychology, 4, 99-105. doi:

$10.1080 / 21683603.2016 .1130582$ 
444 Flake, J. K., Pek, J., \& Hehman, E. (2017). Construct validation in social and personality

445

446

447

448

449

450

451

452

453

454

455

456

457

458

459

460

461

462

463

464

465

466

research: Current practice and recommendations. Social Psychological and Personality Science, 1-9. doi:10.1177/1948550617693063

Fredborg, B., Clark, J., \& Smith, S.D. (2017). An examination of personality traits associated with autonomous sensory meridian response (ASMR). Frontiers in Psychology, 8, 247. doi: $10.3389 /$ fpsyg.2017.00247

IBM Corp. Released 2010. IBM SPSS Statistics for Windows, Version 19.0. Armonk, NY: IBM Corp.

Janik McErlean, A., \& Banissy, M. (2017). Assessing individual variation in personality and empathy traits in self-reported Autonomous Sensory Meridian Response. Multisensory Research, 30, 601-613. doi:10.1163/22134808-00002571

John, O. P., Donahue, E. M., \& Kentle, R. L. (1991). The Big Five Inventory--Versions 4a and 54. Berkeley, CA: University of California, Berkeley, Institute of Personality and Social Research.

John, O. P., \& Srivastava, S. (1999). The Big Five trait taxonomy: History, measurement, and theoretical perspectives. Handbook of personality: Theory and research, 2, 102-138.

Kabat-Zinn, J. (1990). Full catastrophe living: Using the wisdom of your body and mind to face stress, pain, and illness. New York, NY: Dell Publishing.

Lau, M. A., Bishop, S. R., Segal, Z. V., Buis, T., Anderson, N. D., Carlson, L., ... \& Devins, G. (2006). The Toronto mindfulness scale: Development and validation. Journal of clinical psychology, 62, 1445-1467. doi: 10.1002/jclp.20326

Lutz, A., Slagter, H. A., Dunne, J. D., \& Davidson, R. J. (2008). Attention regulation and monitoring in meditation. Trends in Cognitive Sciences, 12, 163-169. 
467 McCrae, R. R., \& John, O. P. (1992). An introduction to the five-factor model and its 468 applications. Journal of Personality, 60, 175-215.

469 Weisberg, Y. J., DeYoung, C. G., \& Hirsh, J. B. (2011). Gender differences in personality across 470 the ten aspects of the Big Five. Frontiers in Psychology, 2, 178.

471 Williams, M.J., \& Dalgleish, T. (2014). Examining the factor structures of the five facet 472 mindfulness questionnaire and the self-compassion scale. Psychological Assessment 26, 473 407-418. doi: 10.1037/a0035566. 


\section{Table $\mathbf{1}$ (on next page)}

Mean Mindful Attention Awareness Scale (MAAS) and Toronto Mindfulness Scale (TMS) scores as Function of Group and Gender (SD in parentheses). 
1 Mean Mindful Attention Awareness Scale (MAAS) and Toronto Mindfulness Scale (TMS)

2 scores as Function of Group and Gender ( $S D$ in parentheses).

\begin{tabular}{ccccc} 
& \multicolumn{2}{c}{ Females } & \multicolumn{2}{c}{ Males } \\
& ASMR & Controls & ASMR & Controls \\
MAAS & $3.40(.794)$ & $3.03(1.17)$ & $3.28(.696)$ & $3.00(1.17)$ \\
TMS - Curiosity & $15.0(5.60)$ & $11.9(6.75)$ & $13.1(5.70)$ & $12.5(5.96)$ \\
TMS - Decentering & $11.3(4.26)$ & $11.6(5.97)$ & $12.3(4.54)$ & $13.2(5.51)$
\end{tabular}

3 


\section{Table 2 (on next page)}

Correlations between the five factors of the ASMR Checklist, Average ASMR tingle intensity ratings $\left(M_{\text {Total }}\right)$, Toronto Mindfulness Scale Curiosity Subscale and Mindful Attention Awareness Scale scores.

TMS-C refers to the Toronto Mindfulness Scale - Curiosity Subscale. MAAS refers to the Mindful Attention Awareness Scale. 
1 Correlations between the five factors of the self-created ASMR Checklist (Fredborg et al.,

2 2017), Average ASMR tingle intensity ratings ( $\left.M_{\text {Total }}\right)$, Toronto Mindfulness Scale -

3 Curiosity Subscale (TMS- C; Lau et al., 2006) and Mindful Attention Awareness Scale

4 (MAAS; Brown \& Ryan, 2003) scores.

\begin{tabular}{|c|c|c|c|c|c|c|c|c|}
\hline & Watching & Touching & $\begin{array}{c}\text { Repetitive } \\
\text { Sounds }\end{array}$ & Simulations & $\begin{array}{l}\text { Mouth } \\
\text { Sounds }\end{array}$ & $M_{\text {Total }}$ & MAAS & TMS-C \\
\hline Watching & 1 & .002 & -0.18 & .001 & -.009 & $.613 * *$ & -.021 & .077 \\
\hline Touching & & 1 & -.005 & .001 & .009 & $.593 * *$ & .115 & $.200 * *$ \\
\hline $\begin{array}{l}\text { Repetitive } \\
\text { Sounds }\end{array}$ & & & 1 & .013 & -.002 & $.344 * *$ & .039 & $.235^{*}$ \\
\hline Simulations & & & & 1 & .011 & $.289 * *$ & $.153 *$ & .090 \\
\hline $\begin{array}{l}\text { Mouth } \\
\text { Sounds }\end{array}$ & & & & & 1 & $.254 * *$ & -.056 & .081 \\
\hline$M_{\text {Total }}$ & & & & & & 1 & .112 & $.160 * *$ \\
\hline MAAS & & & & & & & 1 & $.132 * \mathrm{~b}$ \\
\hline TMS-C & & & & & & & & 1 \\
\hline
\end{tabular}

